\title{
A new way of searching for transients: the ADWO method and its results
}

\section{Zsolt Bagoly*}

Eötvös University, Budapest, Hungary

E-mail: zsolt.bagolydelte.hu

\section{Dorottya Szécsi}

Astronomical Institute, Academy of Sciences of the Czech Republic, Ondřejov, Czech Republic ; School of Physics and Astronomy and Institute of Gravitational Wave Astronomy, University of Birmingham, UK

E-mail: dszecsi@star.sr.bham.ac.uk

\section{Jakub Řípa}

formerly: National Taiwan University, Leung Center for Cosmology and Particle Astrophysics, Taipei, Taiwan

E-mail: ripa.jakub@gmail.com

\section{István I. Racz}

Eötvös University, Budapest, Hungary

E-mail: raczecomplex.elte.hu

\section{István Csabai}

Eötvös University, Budapest, Hungary

E-mail: csabai@complex.elte.hu

\section{László Dobos}

Eötvös University, Budapest, Hungary

E-mail: dobos acomplex.elte.hu

\section{István Horváth}

National University of Public Service, Hungary

E-mail: Horvath. Istvan@uni-nke.hu

\section{Lajos G. Balázs}

Konkoly Observatory, RCAES, Hungarian Academy of Sciences, Hungary

E-mail: lgbalazs@gmail.com

\section{Viktor Tóth}

Eötvös University, Budapest, Hungary

E-mail: tothlviktor@gmail.com 
With the detection of gravitational wave emissions from from merging compact objects, it is now more important than ever to effectively mine the data-set of gamma-satellites for non-triggered, short-duration transients. Hence we developed a new method called the Automatized Detector Weight Optimization (ADWO), applicable for space-borne detectors such as Fermi's GBM and RHESSI's Ge detectors. Provided that the trigger time of an astrophysical event is well known (as in the case of a gravitational wave detection) but the detector response matrix is uncertain, ADWO combines the data of all detectors and energy channels to provide the best signal-tonoise ratio. We used ADWO to successfully identify any potential electromagnetic counterpart of gravitational wave events, as well as to detect previously un-triggered short-duration GRBs in the data-sets.

7th Fermi Symposium 2017

15-20 October 2017

Garmisch-Partenkirchen, Germany

* Speaker. 


\section{Introduction}

The first transient gravitational-wave (GW) signal of GW150914 was observed on 14/09/2015 at 09:50:45.391 UTC by the aLIGO[1]. The Fermi's Gamma-ray Burst Monitor (GBM) custom pipeline looked for prompt gamma-ray counterparts, and found a weak transient source $0.4 \mathrm{~s}$ after the GW event [2]. The INTEGRAL observation of same event was unsuccessful [3], and re-analisys of the GBM data with other methods [4] was unsuccessful too. Currently there are five more aLIGO-aVirgo observations worth to explore: LVT151012, GW121226, GW170104 GW170814 and the GRB170817A detected by the Fermi[5], which had the GW170817 gravitational wave counterpart $1.7 \mathrm{~s}$ earlier, observed by the aLIGO-aVirgo detectors [6]. While the masses of the mergers derived from earlier GW observations were above $20 M_{\odot}$, this last event provided the first direct evidence of a neutron star merger in both the GW and electromagnetic (EM) regimes.

This observation confirms that the standard theoretical model of Short-duration GRBs (SGRBs), namely merging two neutron stars, is viable and results in electromagnetic radiation. But some theories also suggest that a double black hole merger may produce EM signal. E.g. [7] proposed a model where a SGRB is produced by two merging black holes. Here two low-metalicity massive stars are orbiting around each other $[8,9,10]$ with synchronized rotations due to their tight orbit. Their rotational periods are very short (a few days), and these stars evolve homogeneously as the fast rotation prevents them to expand [11]. Assuming that one of the supernova explosions leaves a disk behind, a relativistic jet will be launched during the black holes' merger [12].

Motivated by the detection of a SGRB together with a GW coming from merging neutron stars, as well as by the theoretical possibility that merging black holes may, under the right conditions, also produce SGRBs, we investigated the EM counterparts of all GW triggers-even of those with the higher mass (black hole) progenitors.

\section{The Automatized Detector Weight Optimization (ADWO) method}

The Fermi GBM includes 12 Sodium Iodide ( $\mathrm{NaI}(\mathrm{Tl})$ ) detectors, measuring the EM spectrum from $8 \mathrm{keV}$ to $\sim 1 \mathrm{MeV}$ [13], with two Bismuth Germanate (BGO) scintillation detectors covering the energy range from $\sim 200 \mathrm{keV}$ to $\sim 40 \mathrm{MeV}$.

We also used the available RHESSI data in the case of GW150914. The signal from the $9 \mathrm{Ge}$ detectors is electronically separated into front $(F)$ and rear $(R)$ segments with the energy range of $\sim 30 \mathrm{keV}$ to $\sim 17 \mathrm{MeV}$. Segments F2, F9, R2, R4, R8 and R9 were omitted as they seemed to be malfunctioning.

For a given direction and satellite position the actual detector response matrix (DRM) describes the effective detection area as the function of the incoming photon energy, angular dependence, detector non-linearity and scattering. To calculate the significance of an event, the event's spectral model should be multiplied by the corresponding DRM to obtain photon events, added to the detector's energy-dependent estimated background.

This method cannot be used for signals having large directional errors, as the DRM exhibits a strong angular dependence. Luckily, although the direction is unknown, the time interval where the possible EM counterpart could happen is well determined. To analyze the multi-detector multi-channel continuous data the simplest method would be to sum all the detectors and chan- 
nels. Clearly this is sub-optimal since non-illuminated detectors and energy channels in the noise should be ommited.

We developed the Automatized Detector Weight Optimization (ADWO) method [14], which solves this problem by assigning $e_{i}$ positive normalized weights to the $i^{\text {th }}$ energy channels and the $d_{j}$ positive normalized weights to the $j^{t h}$ detectors. The resulting signal is $S(t)=\sum_{i, j} e_{i} d_{j} C_{i j}(t)$, where $C_{i j}(t)$ denotes the background subtracted lightcurve in the $j$ th detector's $i$ th energy channel. Tuning $e_{i}$ and $d_{j}$ weights ADWO maximizes the Signal's Peak (maximum of $S(t)$ within the search interval) over the Background's Peak (maximum of $S(t)$ outside the search interval). This Signal's Peak over the Background's Peak (SPBPR) is the most important statistical parameters in our analysis. The best weights contain the directional information in the $d_{j}$ part, while the $e_{i}$ values compare the peak luminosity against the background. ADWO provides not only maximum value of SPBPR, but also the exact time of the event. We applied Matlab/Octave's fminsearch routine to find the maximum, the differences of the weights on the final simplex were below $10^{-4}$. ADWO's Matlab/Octave source code is freely available ${ }^{1}$.

The Fermi GBM's continuous time-tagged event (CTTE) data were binned in energy, with limits of 4.4, 12, 27, 50,100, 290, 540, 980 and $2000 \mathrm{keV}\left(e_{1} \ldots e_{8}\right.$, resp.). We used only the upper 6 energy channels, leaving out the noisy hard X-ray region. The RHESSI $100 \mu$ s photon data were binned with the same energy ranges, with two extra $0.98-2$ and 2-17 MeV channels. All the counts were re-binned with an $1 \mathrm{~ms}$ resolution and smoothed by a $64 \mathrm{~ms}$ moving average window.

For the precise background determination one should take the detailed positional information of the satellite and the celestial objects (Earth, Sun, Moon) into account (e.g. [15]). However, here we are only looking for short transients, hence a 6th order polynomial background fit was used as an exact model for the background. ADWO assume no structure in the background region, therefore background regions with peaks (charged particle signals in one detector, etc.) should be excluded. Singular $e_{i} / d_{j}$ values will hint such events. Here the backgrounds around the GW events contains no such peaks.

\section{Transient search results}

We applied the ADWO method with a $6 \mathrm{~s}$ long signal window in the $(-195,495)$ s full interval relative to the GW150914 event. Applied on the Fermi CTTE data ADWO obtained SPBPR=1.91, $474 \mathrm{~ms}$ after the trigger[14]. Using the observed data $10^{4} \mathrm{MC}$ simulations were made producing 86 cases with higher SPBPR. Hence the the false alarm rate of was determined to be $\approx 1.4 \mathrm{mHz}$, and the false alarm probability of $\approx 7.5 \cdot 10^{-3}$, which is higher than the $2.2 \cdot 10^{-3}$ value given by [16]. On the RHESSI data ADWO obtained a maximum of SPBPR=1.322, $2.879 \mathrm{~ms}$ after the $\mathrm{GW}$ trigger, well in the fluctuation range.

We also analyzed of LVT151012[14], covering the $(-195,495) \mathrm{s}$ window around the trigger. ADWO produced a SPBPR=1.805, $652 \mathrm{~ms}$ after the GW event (Fig. 2), with a MC false alarm probability of $\approx 3.7 \cdot 10^{-2}$.

ADWO was applied for the GW151226 event, in the $(-195,495) \mathrm{s}$ window around the trigger. The method got a relatively low peak with $\mathrm{SPBPR}=1.321,1950 \mathrm{~ms}$ before the $\mathrm{GW}$ trigger. The SPBPR value is so low that it cannot be considered as a real signal.

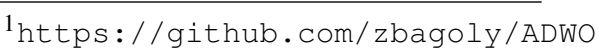



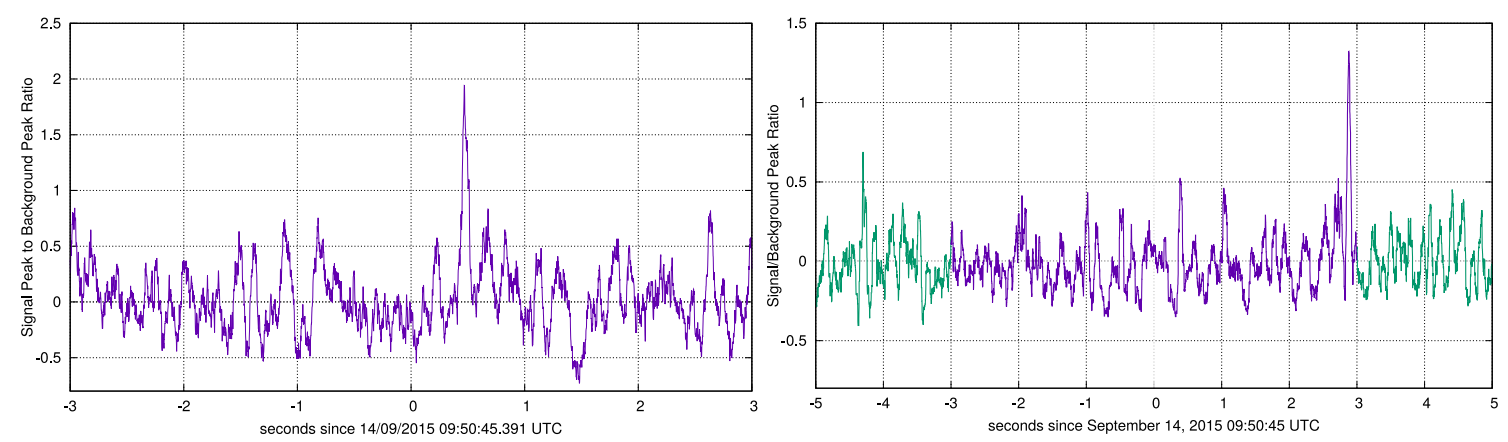

Figure 1: ADWO lightcurves of Fermi (left) and RHESSI (right) data around the GW150914 trigger. The green part shows the background, i.e. ADWO searches inside the $[-3 ; 3]$ s region for best signal.
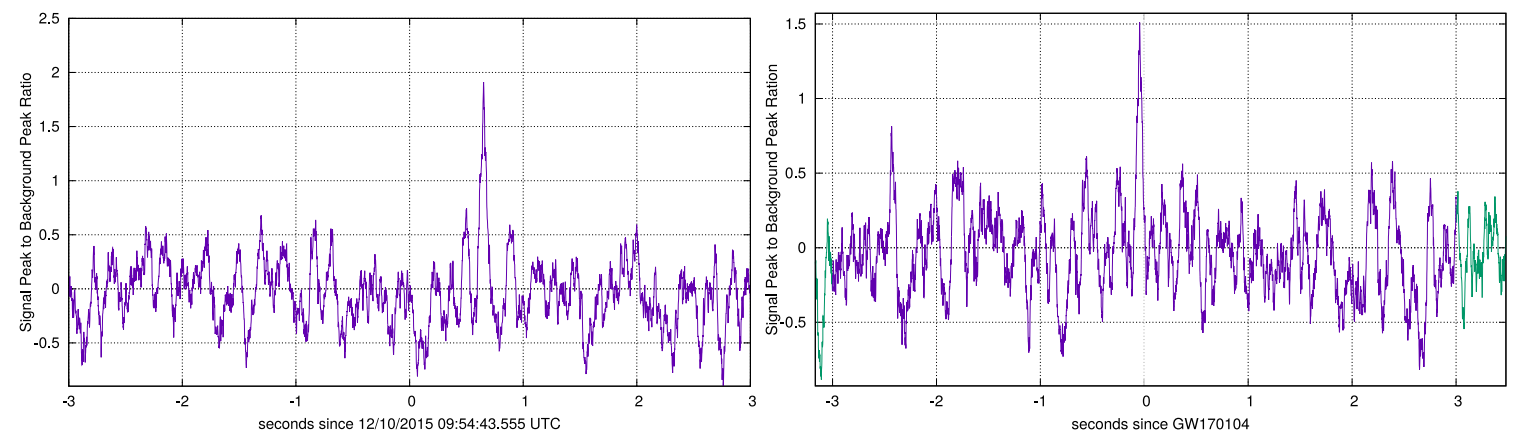

Figure 2: ADWO analysis of LVT151012 and GW170104, in the 27-2000 keV range.

ADWO was also applied for the GW170104 event too, with the $(-200,140)$ s interval around the trigger. A maximum of SPBPR=1.51 at $T \approx-50 \mathrm{~ms}$ was obtained, which is probably noise (Fig. 2). No correlation is visible with the Agile MCAL's E2 and E3 peaks [17], not even when a smaller $1 \mathrm{~s}$ wide search window was centered directly on the events.

The GW170814 event's Fermi GBM data were analyzed with ADWO in the $(-50,250)$ s interval (there was a particle event around $-50 \mathrm{~s}$ ). The SPBPR $=1.28$ maximum is probably a fluctuation. For the GRB170817A/GW170817 merger event GBM data was analyzed in the $(-100,100) \mathrm{s}$ interval (limited by the spacecraft's SAA entry). ADWO provided a strong signal with a maximum of SPBPR=2.6902, at the same position as GRB170817A was observed [5].

\section{Discussion}

We repeated ADWO on the whole day 61.4 ks GBM data on 15/09/2015. The SPBPR values of these $10235 \times 6 \mathrm{~s}$ window slices with the corresponding sum of the $27-290 \mathrm{keV}$ weights are plotted on Fig. 3. Furthermore, we analyzed all the GRBs triggered by Fermi with $T_{90}<10$ s. The sum of the $50-290 \mathrm{keV}$ weights (softness ratio, calculated around the signal peak) and the SPBPR values are also plotted events having SPBPR below 6. Most of the (triggered) GRBs have higher than 0.5 peak softness. Compared with them, GRB170817A/GW170817 shows almost a "tipical" peak softness and SPBPR.

One can see that several GBM daily background slices have a spectrally hard peak with high SPBPR, well in the GRB area. Although most of them produced by fluctuation, we have to em- 
phasis that untriggered EM events (e.g. untriggered GRBs) should expected to be in the same area. Three background slices have even higher SPBPR value than GRB150522B, with much harder peak spectrum. The detailed analysis of the untriggered GRBs (e.g. GRB150921153 and GRB160301788 based on [18, 19], on Fig. 3.) is subject of our future work. ADWO sensitivity depends on the peak flux intensity and not on the overall shape of the signal: $\approx 20 \%$ of the $T_{90} \leq 10 \mathrm{~s}$ Fermi SGRBs show smaller SPBPR values than GW150914 (Fig. 3.).

As our ADWO method is independently developed, and only relies on the raw data of the satellite, it can provide a strong, independent test to any future EM signal. We therefore expect that ADWO will successfully identify any potential electromagnetic counterpart of a given trigger (e.g. GW events), as well as to detect previously un-triggered short-duration GRBs in the data-sets.

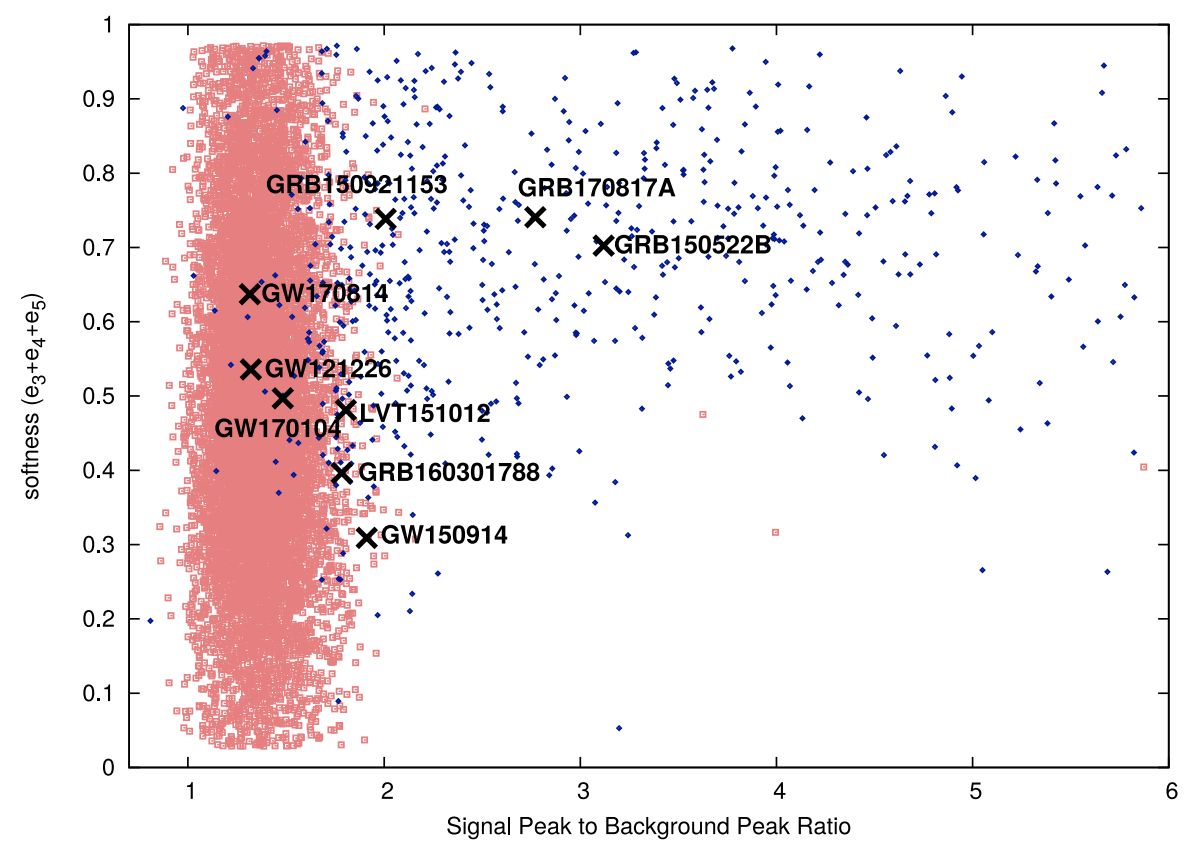

Figure 3: Signal Peak to Background Peak Ratio and peak softness (the sum of the $50-290 \mathrm{keV}$ weights) for the $61.4 \mathrm{ks}$ GBM data background (red open squares) and for the $T_{90} \leq 10 \mathrm{~s}$ Fermi SGRBs (small crosses). The transients mentioned in the article are also shown.

\section{Acknowledgements}

This work was supported by OTKA grants NN111016 and NN114560. D. Sz. was partially supported by STFC and by the Czech grants 13-10589S and 16-01116S GA ČR.

\section{References}

[1] B. P. Abbott, R. Abbott, T. D. Abbott, M. R. Abernathy, F. Acernese, K. Ackley et al., Observation of Gravitational Waves from a Binary Black Hole Merger, Physical Review Letters 116 (Feb., 2016) 061102, [1602.03837].

[2] V. Connaughton, E. Burns, A. Goldstein, L. Blackburn, M. S. Briggs, B.-B. Zhang et al., Fermi GBM Observations of LIGO Gravitational-wave Event GW150914, ApJL 826 (July, 2016) L6, [1602.03920]. 
[3] V. Savchenko, C. Ferrigno, S. Mereghetti, L. Natalucci, A. Bazzano, E. Bozzo et al., INTEGRAL Upper Limits on Gamma-Ray Emission Associated with the Gravitational Wave Event GW150914, ApJL 820 (Apr., 2016) L36, [1602 . 04180 ].

[4] J. Greiner, J. M. Burgess, V. Savchenko and H.-F. Yu, On the Fermi-GBM Event 0.4 s after GW150914, ApJL 827 (Aug., 2016) L38, [1606. 00314 ].

[5] A. Goldstein, P. Veres, E. Burns, M. S. Briggs, R. Hamburg, D. Kocevski et al., An ordinary short gamma-ray burst with extraordinary implications: Fermi -gbm detection of grb 170817 a, The Astrophysical Journal 848 (10, 2017) L14.

[6] Ligo Scientific Collaboration and Virgo Collaboration collaboration, B. P. Abbott, R. Abbott, T. D. Abbott, F. Acernese, K. Ackley, C. Adams et al., Gw170817: Observation of gravitational waves from a binary neutron star inspiral, Phys. Rev. Lett. 119 (Oct, 2017) 161101.

[7] R. Perna, D. Lazzati and B. Giacomazzo, Short Gamma-Ray Bursts from the Merger of Two Black Holes, ApJL 821 (Apr., 2016) L18, [1602.05140].

[8] S. E. de Mink, M. Cantiello, N. Langer, O. R. Pols, I. Brott and S.-C. Yoon, Rotational mixing in massive binaries. Detached short-period systems, A\&A 497 (Apr., 2009) 243-253, [0 902.1751 ].

[9] P. Marchant, N. Langer, P. Podsiadlowski, T. M. Tauris and T. J. Moriya, A new route towards merging massive black holes, A\&A 588 (Apr., 2016) A50, [1601.03718].

[10] I. Mandel and S. E. de Mink, Merging binary black holes formed through chemically homogeneous evolution in short-period stellar binaries, MNRAS 458 (May, 2016) 2634-2647, [1601. 00007 ].

[11] D. Szécsi, N. Langer, S.-C. Yoon, D. Sanyal, S. de Mink, C. J. Evans et al., Low-metallicity massive single stars with rotation. Evolutionary models applicable to I Zwicky 18, A\&A 581 (Sept., 2015) A15, [1506.09132].

[12] D. Szécsi, How may short-duration GRBs form? A review of progenitor theories., Contributions of the Astronomical Observatory Skalnate Pleso 47 (July, 2017) 108-115, [1710. 05356].

[13] C. Meegan, G. Lichti, P. N. Bhat, E. Bissaldi, M. S. Briggs, V. Connaughton et al., The Fermi Gamma-ray Burst Monitor, ApJ 702 (Sept., 2009) 791-804, [0 908.0450 ].

[14] Z. Bagoly, D. Szécsi, L. G. Balázs, I. Csabai, I. Horváth, L. Dobos et al., Searching for electromagnetic counterpart of LIGO gravitational waves in the Fermi GBM data with ADWO, A\&A 593 (Sept., 2016) L10, [1603.06611].

[15] D. Szécsi, Z. Bagoly, J. Kóbori, I. Horváth and L. G. Balázs, Direction dependent background fitting for the Fermi GBM data, A\&A 557 (Sept., 2013) A8, [1306.3812].

[16] V. Connaughton, E. Burns, A. Goldstein, M. S. Briggs, B.-B. Zhang, C. M. Hui et al., Fermi GBM Observations of LIGO Gravitational Wave event GW150914, Accepted for publication in ApJL, ArXiv e-prints (Feb., 2016), [1602.03920].

[17] F. Verrecchia, M. Tavani, A. Ursi, A. Argan, C. Pittori, I. Donnarumma et al., AGILE Observations of the Gravitational-wave Source GW170104, ApJL 847 (Oct., 2017) L20, [1 706.00029 ].

[18] K. Siellez, G. Carullo, S. Forsyth, L. Cadonati, M. Briggs and V. Connaughton, The Untriggered GRBs from Fermi-GBM in Coincidence with LIGO, LPI Contributions 1962 (Oct., 2016) 4103.

[19] M. S. Briggs, R. Hamburg, P. Veres, E. Burns, C. M. Hui, V. Connaughton et al., Detecting Fainter Short GRBs with the Fermi Gamma-Ray Burst Monitor, LPI Contributions 1962 (Oct., 2016) 4097. 